\title{
ON THE DISTINCTION BETWEEN THE CLASSES OF DIXMIER AND CONNES-DIXMIER TRACES
}

\author{
FEDOR SUKOCHEV, ALEXANDR USACHEV, AND DMITRIY ZANIN \\ (Communicated by Varghese Mathai)
}

\begin{abstract}
We prove that the classes of Dixmier and Connes-Dixmier traces differ even on the Dixmier ideal $\mathcal{M}_{1, \infty}$. We construct a Marcinkiewicz space $\mathcal{M}_{\psi}$ and a positive operator $T \in \mathcal{M}_{\psi}$ which is Connes-Dixmier measurable but which is not Dixmier measurable.
\end{abstract}

\section{INTRODUCTION AND PRELIMINARES}

In [5] J. Dixmier proved that there exists a non-normal trace (a Dixmier trace) on the non-commutative Marcinkiewicz spaces $\mathcal{M}_{\psi}$ for every $\psi$ such that

$$
\lim _{t \rightarrow \infty} \frac{\psi(2 t)}{\psi(t)}=1
$$

In [4] A. Connes introduced a subclass of Dixmier traces, later termed in 9] ConnesDixmier traces. In this paper, we investigate the relationship between these two classes and show that they differ even on the classical Dixmier ideal $\mathcal{M}_{1, \infty}$. Furthermore, we prove that there is a Marcinkiewicz ideal $\mathcal{M}_{\psi}$, with $\psi$ satisfying (1.1) such that these two classes of traces generate distinct sets of measurable elements (see [4, IV.2. $\beta$.Definition 7] and Definitions [1.3 and [1.4 below).

1.1. Generalized limits. Let $l_{\infty}$ be the Banach space of all bounded sequences $x=\left(x_{0}, x_{1}, \ldots\right)$ with the norm

$$
\|x\|_{l_{\infty}}:=\sup _{n \geq 0}\left|x_{n}\right|
$$

A normalized positive linear functional on $l_{\infty}$ which equals the ordinary limit on convergent sequences is called a generalized limit. For every $n \in \mathbb{N}$ we define a dilation operator $\sigma_{n}: l_{\infty} \rightarrow l_{\infty}$ as follows:

$$
\sigma_{n}\left(x_{0}, x_{1}, \ldots\right)=(\underbrace{x_{0}, \ldots, x_{0}}_{n}, \underbrace{x_{1}, \ldots, x_{1}}_{n}, \ldots) .
$$

If a generalized limit $\omega$ on $l_{\infty}$ satisfies the condition

$$
\omega\left(\sigma_{n} x\right)=\omega(x)
$$

for every $x \in l_{\infty}$ and any $n \in \mathbb{N}$, then $\omega$ is called a dilation invariant generalized limit.

Received by the editors September 22, 2011.

2010 Mathematics Subject Classification. Primary 58B34, 46L52.

Key words and phrases. Dixmier trace, Marcinkiewicz space, generalized limits.

The authors' research was supported by the Australian Research Council. 
Let $L_{\infty}=L_{\infty}(0, \infty)$ be the space of all real-valued bounded Lebesgue measurable functions on $(0, \infty)$ equipped with the norm

$$
\|x\|_{L_{\infty}}:=\operatorname{ess~sup}_{t>0}|x(t)| .
$$

A normalized positive linear functional on $L_{\infty}$ which equals the ordinary limit on convergent (at infinity) sequences is called a generalized limit. For every $x \in L_{\infty}$ and for any generalized limit $\gamma$ on $L_{\infty}$ the following inequalities hold:

$$
\liminf _{t \rightarrow \infty} x(t) \leq \gamma(x) \leq \limsup _{t \rightarrow \infty} x(t) .
$$

By the Hahn-Banach extension theorem, for every $x \in L_{\infty}$ there exist generalized limits $\gamma_{1}$ and $\gamma_{2}$ such that

$$
\gamma_{1}(x)=\limsup _{t \rightarrow \infty} x(t), \quad \gamma_{2}(x)=\liminf _{t \rightarrow \infty} x(t) .
$$

We define a dilation operator $\sigma_{s}: L_{\infty} \rightarrow L_{\infty}$ as follows:

$$
\left(\sigma_{s} x\right)(t)=x(t / s), \quad s>0 .
$$

A generalized limit $\omega$ on $L_{\infty}$ is said to be dilation invariant if

$$
\omega\left(\sigma_{s} x\right)=\omega(x)
$$

for every $x \in L_{\infty}$ and any $s>0$.

Let $\pi$ be the isometric embedding $\pi: l_{\infty} \rightarrow L_{\infty}$ given by

$$
\left\{x_{n}\right\}_{n=0}^{\infty} \stackrel{\pi}{\mapsto} \sum_{n=0}^{\infty} x_{n} \chi_{(n, n+1]} .
$$

The following natural way to generate dilation invariant generalized limits was suggested in [4, Section IV, $2 \beta]$. A. Connes observed that for any generalized limit $\gamma$ on $L_{\infty}$ a functional $\omega:=\gamma \circ M \circ \pi$ is a dilation invariant generalized limit on $l_{\infty}$. Here, the bounded operator $M: L_{\infty} \rightarrow L_{\infty}$ is given by the formula

$$
(M x)(t):=\frac{1}{\log t} \int_{1}^{t} x(s) \frac{d s}{s} .
$$

Throughout the paper we denote by $\log t$ the natural logarithm and by $\log _{2} t$ the logarithm with base 2 .

1.2. Marcinkiewicz spaces. Let $B(H)$ be an algebra of all bounded linear operators on a separable Hilbert space $H$ equipped with the uniform norm and let $\mathrm{Tr}$ be the standard trace.

For every operator $T \in B(H)$ a generalized singular value function $\mu(T)$ is defined by the formula

$$
\mu(t, T)=\inf \{\|T p\|: p \text { is a projection in } B(H) \text { with } \operatorname{Tr}(1-p) \leq t\} .
$$

For a compact operator $T$, it can be proven that $\mu(k, T)$ is the $k$-th largest eigenvalue of an operator $|T|, k \geq 0$.

Since $B(H)$ is an atomic von Neumann algebra and traces of all atoms are equal to 1 , it follows that $\mu(T)$ is a step function and $\mu(T)=\pi(\mu(k, T))$ for every $T \in B(H)$.

Let $\Omega$ denote the set of all concave functions $\psi:[0, \infty) \rightarrow[0, \infty)$ such that $\lim _{t \rightarrow 0+} \psi(t)=0$ and $\lim _{t \rightarrow \infty} \psi(t)=\infty$. 
Let $\psi \in \Omega$. Consider the Banach ideal $\left(\mathcal{M}_{\psi},\|\cdot\|_{\mathcal{M}_{\psi}}\right)$ of compact operators in $B(H)$ given by (see e.g. [2, 8, 9])

$$
\mathcal{M}_{\psi}:=\left\{T:\|T\|_{\mathcal{M}_{\psi}}:=\sup _{n \geq 0} \frac{1}{\psi(1+n)} \sum_{k=0}^{n} \mu(k, T)<\infty\right\} .
$$

For $f \in L_{\infty}$ we set

$$
a(t, f):=\frac{1}{\psi(t)} \int_{0}^{t} f^{*}(s) d s,
$$

where $f^{*}$ denotes the decreasing rearrangement of the function $|f|$, that is,

$$
f^{*}(t):=\inf \{s \geq 0: \operatorname{meas}(\{|f|>s\}) \leq t\} .
$$

We define the Marcinkiewicz function space $M_{\psi}$ of real-valued measurable functions $f$ on $(0, \infty)$ by setting

$$
\|f\|_{M_{\psi}}:=\sup _{t>0} a(t, f)<\infty .
$$

For a compact operator we have $T \in \mathcal{M}_{\psi}$ if and only if $\mu(T) \in M_{\psi}$.

In the case when $\psi(t)=\log (1+t)$ the space $\mathcal{M}_{\psi}$ is a well-known Dixmier ideal $\mathcal{M}_{1, \infty}$.

1.3. Singular traces on general Marcinkiewicz spaces. For an arbitrary dilation invariant generalized limit $\omega$ on $l_{\infty}$ the weight

$$
\operatorname{Tr}_{\omega}(T):=\omega\left(\left\{\frac{1}{\log (2+n)} \sum_{k=0}^{n} \mu(k, T)\right\}_{n=0}^{\infty}\right), \quad 0 \leq T \in \mathcal{M}_{1, \infty},
$$

extends to a non-normal trace (a Dixmier trace) on $\mathcal{M}_{1, \infty}$ [5, 4, 2, We denote the set of all Dixmier traces by $\mathcal{D}$.

The subclass $\mathcal{C} \subset \mathcal{D}$ of all Dixmier traces $\operatorname{Tr}_{\omega}$ defined by $\omega=\gamma \circ M \circ \pi$ was termed Connes-Dixmier traces in 9 . A priori, $\mathcal{C} \subseteq \mathcal{D}$ and the question about the precise relationship between these two classes arises naturally. Recently the distinction between $\mathcal{C}$ and $\mathcal{D}$ was studied by A. Pietsch in terms of density characters (see [1][13]). For the discussion of various classes of singular traces, we refer to [1, 2, 10].

The first main result of the present paper (Theorem 2.2 below) shows that the inclusion $\mathcal{C} \subset \mathcal{D}$ is proper. Our approach is completely different from that of A. Pietsch and the proof provided here is much shorter.

It has become traditional to reduce various problems about Dixmier traces to its commutative analogues.

For every dilation invariant generalized limit $\omega$ on $L_{\infty}$ one can define a commutative analogue of the Dixmier trace (a Dixmier functional on $M_{1, \infty}$ ) as follows:

$$
\tau_{\omega}(f)=\omega(a(t, f)), 0 \leq f \in M_{1, \infty}
$$

and extend it to $M_{1, \infty}$ by linearity.

It was shown in [7, 8, that, for a general Marcinkiewicz space $\mathcal{M}_{\psi}$, the following conditions are equivalent:

(i) The space $\mathcal{M}_{\psi}$ admits non-trivial Dixmier traces.

(ii) The function $\psi \in \Omega$ satisfies the condition

$$
\liminf _{t \rightarrow \infty} \frac{\psi(2 t)}{\psi(t)}=1
$$


(iii) There exists a dilation invariant limit $\omega$ on $l_{\infty}$ such that

$$
\omega\left(\frac{\psi(2 n)}{\psi(n)}\right)=1
$$

It was also proven in [8, Proposition 9, Theorem 11] that for $\psi \in \Omega$ satisfying (1.4), the weight

$$
\operatorname{Tr}_{\omega}(T):=\omega\left(\left\{\frac{1}{\psi(1+n)} \sum_{k=0}^{n} \mu(k, T)\right\}_{n=0}^{\infty}\right), \quad 0 \leq T \in \mathcal{M}_{\psi},
$$

extends to a Dixmier trace on $\mathcal{M}_{\psi}$ if and only if a dilation invariant generalized limit $\omega$ on $l_{\infty}$ satisfies (1.5).

Similarly to the definition of Connes-Dixmier traces on $\mathcal{M}_{1, \infty}$, for every $\psi \in \Omega$ satisfing (1.4) and any dilation invariant limit $\omega=\gamma \circ M \circ \pi$ on $l_{\infty}$ satisfying (1.5) we can define a Connes-Dixmier trace $\operatorname{Tr}_{\omega}$ on $\mathcal{M}_{\psi}$.

Similarly to (1.3), we define Dixmier and Connes-Dixmier functionals $\tau_{\omega}$ for every dilation invariant generalized limit $\omega$ on $L_{\infty}$ satisfying

$$
\omega\left(\frac{\psi(2 t)}{\psi(t)}\right)=1
$$

Remark 1.1. By [16, Theorem 14, Corollary 15] and [8, Theorem 11] we know that for every Dixmier trace $\operatorname{Tr}_{\omega_{1}}$ on $\mathcal{M}_{\psi}$ ( $\omega_{1}$ is a dilation invariant generalized limit on $\left.l_{\infty}\right)$ there exists a Dixmier functional $\tau_{\omega_{2}}$ on $M_{\psi}\left(\omega_{2}\right.$ is a dilation invariant generalized limit on $L_{\infty}$ ) such that

$$
\operatorname{Tr}_{\omega_{1}}(T)=\tau_{\omega_{2}}(\mu(T)), \quad 0 \leq T \in \mathcal{M}_{\psi} .
$$

The converse implication also holds.

The following lemma was borrowed from [8, Proposition 9].

Lemma 1.2. Let $\psi \in \Omega$ satisfy (1.4) and let $\omega$ be a dilation invariant generalized limit on $L_{\infty}$ satisfying (1.6). For every $f \in M_{\psi}$, we have

$$
\omega\left(\frac{t f^{*}(t)}{\psi(t)}\right)=0
$$

Proof. Since $\omega$ is a dilation invariant generalized limit,

$$
\begin{aligned}
\omega\left(\frac{1}{\psi(2 t)} \int_{0}^{2 t} f^{*}(s) d s\right) & =\omega\left(\frac{1}{\psi(t)} \int_{0}^{t} f^{*}(s) d s\right) \\
& =\omega\left(\frac{\psi(2 t)}{\psi(t)} \frac{1}{\psi(2 t)} \int_{0}^{t} f^{*}(s) d s\right) .
\end{aligned}
$$

Since $\omega$ satisfies (1.6), it follows from [8, Proposition 4] that

$$
\omega\left(\frac{1}{\psi(2 t)} \int_{0}^{2 t} f^{*}(s) d s\right)=\omega\left(\frac{1}{\psi(2 t)} \int_{0}^{t} f^{*}(s) d s\right) .
$$

Hence,

and, furthermore,

$$
\omega\left(\frac{1}{\psi(2 t)} \int_{t}^{2 t} f^{*}(s) d s\right)=0
$$

$$
\omega\left(\frac{2 t f^{*}(2 t)}{\psi(2 t)}\right)=0
$$


Again, applying dilation invariance of $\omega$, we have

$$
\omega\left(\frac{t f^{*}(t)}{\psi(t)}\right)=0
$$

1.4. Measurability. The following definitions were motivated by A. Connes 4 , IV.2. $\beta$.Definition 7] (see also [9, Definition 3.2]) in the case when $\psi(t)=\log (1+t)$.

Definition 1.3. Let $\psi \in \Omega$ satisfy (1.4). An operator $T \in \mathcal{M}_{\psi}$ is called Dixmier measurable if $\operatorname{Tr}_{\omega}(T)$ takes the same value for all $\operatorname{Tr}_{\omega} \in \mathcal{D}$.

Definition 1.4. Let $\psi \in \Omega$ satisfy (1.4). An operator $T \in \mathcal{M}_{\psi}$ is called ConnesDixmier measurable if $\operatorname{Tr}_{\omega}(T)$ takes the same value for all $\operatorname{Tr}_{\omega} \in \mathcal{C}$.

Theorem 1.5 (Corollary 3.9 from [9]). If $\psi \in \Omega$ satisfying (1.4) is such that

$$
t \cdot \frac{d}{d t} \log \left(\psi\left(e^{t}\right)\right)<C
$$

for some $C>0$ and for all $t>0$, then for a positive operator $T \in \mathcal{M}_{\psi}$ the following statements are equivalent:

(i) $T$ is Dixmier measurable.

(ii) $T$ is Connes-Dixmier measurable.

(iii) There exists

$$
\lim _{n \rightarrow \infty} \frac{1}{\psi(n+1)} \sum_{k=0}^{n} \mu(k, T) .
$$

It is easy to check that the function $\psi(t)=\log (1+t)$ satisfies the condition (1.8). Notwithstanding the difference between the sets of Dixmier and Connes-Dixmier traces, a positive operator $T \in \mathcal{M}_{1, \infty}$ is Connes-Dixmier measurable if and only if it is Dixmier measurable.

This result naturally raises the question, whether for an arbitrary function $\psi \in \Omega$ satisfying (1.4) the Connes-Dixmier measurability is equivalent to Dixmier measurability on the cone of all positive elements from $\mathcal{M}_{\psi}$. Our second main result (Theorem 3.4 below) shows that the answer is (surprisingly) negative.

An example of the function $\psi \in \Omega$ satisfying (1.4) but failing the equivalence (i) $\Leftrightarrow$ (iii) was constructed in [6, Theorem 4.6]. However, if $\psi \in \Omega$ satisfies (1.1), then Theorem 3.3 below shows that the equivalence (i) $\Leftrightarrow$ (iii) holds independently of the condition (1.8).

\section{The Classes of DiXmier and Connes-DiXmier traces are Distinct}

Denote by $\mathcal{M}_{\psi}^{0}$ the separable part of the space $\mathcal{M}_{\psi}$, that is, the closure in $\mathcal{M}_{\psi}$ of the set of all finite-dimensional operators from $B(H)$. The following lemma was proven in [9, Theorems 2.8 and 5.12] (see also [2, Theorems 7.3 and 7.4]).

Lemma 2.1. If $\psi \in \Omega$ satisfies (1.1), then

$$
\operatorname{dist}\left(T, \mathcal{M}_{\psi}^{0}\right)=\sup _{\operatorname{Tr}_{\omega} \in \mathcal{D}} \operatorname{Tr}_{\omega}(T), \quad 0 \leq T \in \mathcal{M}_{\psi} .
$$

If $\psi$ satisfies (1.8), then there exists $c>1$ such that

$$
\sup _{\operatorname{Tr}_{\omega} \in \mathcal{C}} \operatorname{Tr}_{\omega}(T) \leq \operatorname{dist}\left(T, \mathcal{M}_{\psi}^{0}\right) \leq c \cdot \sup _{\operatorname{Tr}_{\omega} \in \mathcal{C}} \operatorname{Tr}_{\omega}(T), \quad 0 \leq T \in \mathcal{M}_{\psi} .
$$


In view of the difference between (2.1) and (2.2), the following question arises naturally: "Is the constant $c$ in (2.2) necessarily strictly greater than 1?" The following theorem shows that the inclusion $\mathcal{C} \subset \mathcal{D}$ is proper and answers this question in the affirmative.

Theorem 2.2. There exists a positive operator $T_{0} \in \mathcal{M}_{1, \infty}$ such that

$$
\sup _{\operatorname{Tr}_{\omega} \in \mathcal{D}} \operatorname{Tr}_{\omega}\left(T_{0}\right)>\sup _{\operatorname{Tr}_{\omega} \in \mathcal{C}} \operatorname{Tr}_{\omega}\left(T_{0}\right) .
$$

Proof. Let $T_{0}$ be such that

$$
\mu\left(T_{0}\right)=\sup _{k \geq 0} 2^{k-2^{k}} \chi_{\left[0,2^{2^{k}}\right)} .
$$

We set $f_{0}=\mu\left(T_{0}\right)$. By Lemma 2.1, we have

$$
\begin{aligned}
\sup _{\operatorname{Tr}_{\omega} \in \mathcal{D}} \operatorname{Tr}_{\omega}\left(T_{0}\right) & =\operatorname{dist}\left(T, \mathcal{M}_{\psi}^{0}\right) \\
& =\limsup _{t \rightarrow \infty} a\left(t, f_{0}\right) .
\end{aligned}
$$

By (1.2), we have

$$
\sup _{\operatorname{Tr}_{\omega} \in \mathcal{C}} \operatorname{Tr}_{\omega}\left(T_{0}\right)=\limsup _{t \rightarrow \infty}\left(M a\left(\cdot, f_{0}\right)\right)(t) .
$$

So, it is sufficient to prove that

$$
\limsup _{t \rightarrow \infty} a\left(t, f_{0}\right)>\limsup _{t \rightarrow \infty}\left(M a\left(\cdot, f_{0}\right)\right)(t) .
$$

Clearly, $f_{0}=f_{0}^{*}$. For every $2^{2^{n}} \leq t<2^{2^{n+1}}$ we have

$$
\begin{aligned}
a\left(t, f_{0}\right) & =\frac{1}{\log (1+t)}\left(\int_{0}^{2^{2^{n}}} f_{0}(s) d s+\left(t-2^{2^{n}}\right) f_{0}(t)\right) \\
& =\frac{1}{\log (1+t)}\left(\sum_{k=1}^{n} \int_{2^{2^{k-1}}}^{2^{2^{k}}} 2^{k-2^{k}} d s+t f_{0}(t)+O(1)\right) \\
& =\frac{2^{n+1}}{\log t}+\frac{t f_{0}^{*}(t)}{\log (1+t)}+o(1) .
\end{aligned}
$$

It is easy to check that $f_{0} \in M_{1, \infty}$ and, hence, $T_{0} \in \mathcal{M}_{1, \infty}$.

By Lemma 1.2 $\gamma \circ M\left(\frac{t f_{0}^{*}(t)}{\log (1+t)}\right)=0$ for every generalized limit $\gamma$ on $L_{\infty}$ and, appealing to (1.2), we conclude that

$$
\lim _{t \rightarrow \infty} M\left(\frac{s f_{0}^{*}(s)}{\log (1+s)}\right)(t)=0, \text { for every } f \in M_{1, \infty}
$$

Define the function $x \in L_{\infty}$ by setting

$$
x(t):=\sum_{n=0}^{\infty} \frac{2^{n}}{\log t} \chi_{\left[2^{2^{n}}, 2^{2}\right.}(t) \quad t>0 .
$$


Hence, we obtain from (2.3) and (2.4),

$$
\limsup _{t \rightarrow \infty}\left(M a\left(\cdot, f_{0}\right)\right)(t)=2 \limsup _{t \rightarrow \infty}(M x)(t) .
$$

For $2^{2^{n}} \leq t<2^{2^{n+1}}$, we have

$$
(M x)(t)=\frac{1}{\log t}\left(\sum_{k=0}^{n-1} 2^{k} \int_{2^{2^{k}}}^{2^{2^{k+1}}} \frac{d \log s}{\log s}+2^{n} \int_{2^{2^{n}}}^{t} \frac{d \log s}{\log s}+O(1)\right) .
$$

Since

$$
\int \frac{d \log s}{\log s}=\frac{\log _{2}\left(\log _{2} s\right)-\log _{2}\left(\log _{2} e\right)}{\log _{2} e}+C
$$

it follows that

$$
\begin{aligned}
(M x)(t) & =\frac{\log 2}{\log t}\left(\sum_{k=0}^{n-1} 2^{k}+2^{n}\left(\log _{2}\left(\log _{2} t\right)-n\right)\right)+O(1) \\
& =\frac{2^{n} \log 2}{\log t}\left(1+\log _{2}\left(\log _{2} t\right)-n\right)+o(1) .
\end{aligned}
$$

The function

$$
g: t \rightarrow \frac{2^{n} \log 2}{\log t}\left(1+\log _{2}\left(\log _{2} t\right)-n\right), \quad t \in\left[2^{2^{n}}, 2^{2^{n+1}}\right)
$$

has extrema at

$$
t_{n}=2^{2^{\frac{1}{\log 2}-1+n}} \in\left[2^{2^{n}}, 2^{2^{n+1}}\right), n \in \mathbb{N} .
$$

We have $g\left(t_{n}\right)=\frac{2}{e \log 2}$ for every $n \in \mathbb{N}$. Since $g\left(2^{2^{n}}\right)=1$ for every $n \in \mathbb{N}$ and since $g$ is continuous on $(1, \infty)$, it follows that $\lim \sup _{t \rightarrow \infty} g(t)=\frac{2}{e \log 2}$ and

$$
\limsup _{t \rightarrow \infty}\left(M a\left(\cdot, f_{0}\right)\right)(t)=2 \limsup _{t \rightarrow \infty}(M x)(t)=\frac{4}{e \log 2} .
$$

By the definition we have $f_{0}\left(2^{2^{n}}\right)=2^{n+1-2^{n+1}}$, and so, from (2.3), we obtain

$$
\begin{aligned}
\limsup _{t \rightarrow \infty} a\left(t, f_{0}\right) & \geq \limsup _{n \rightarrow \infty} a\left(2^{2^{n}}, f_{0}\right) \\
& =\frac{1}{\log 2} \cdot \limsup _{n \rightarrow \infty}\left(\frac{2^{n+1}}{\log _{2} 2^{2^{n}}}+\frac{2^{2^{n}} f_{0}\left(2^{2^{n}}\right)}{\log _{2} 2^{2^{n}}}\right) \\
& =\frac{1}{\log 2} \cdot \limsup _{n \rightarrow \infty}\left(2+\frac{2^{2^{n}} 2^{n+1-2^{n+1}}}{2^{n}}\right) \\
& =\frac{2}{\log 2} \\
& >\frac{4}{e \log 2} \\
& =\limsup _{t \rightarrow \infty}\left(\operatorname{Ma}\left(\cdot, f_{0}\right)\right)(t) .
\end{aligned}
$$




\section{The Classes of Dixmier and Connes-Dixmier MEASURABLE ELEMENTS ARE DISTINCT}

The following lemma is taken from [15] (see Theorem 18 or [14, Theorem 6.1.3]).

Lemma 3.1. Let $x \in L_{\infty}$ such that $x \circ \exp$ is uniformly continuous. The equality $\omega(x)=A$ holds for every dilation invariant generalized limit $\omega$ on $L_{\infty}$ if and only if

$$
\lim _{t \rightarrow \infty} \frac{1}{\log t} \int_{1}^{t} x(\alpha s) \frac{d s}{s}=A
$$

uniformly in $\alpha \geq 1$.

Corollary 3.2. Let $\psi \in \Omega$ satisfy (1.4). Let $f \in M_{\psi}$ and let $A$ be a real number. The equality $\tau_{\omega}(f)=A$ holds for every Dixmier functional $\tau_{\omega}$ if and only if

$$
\lim _{t \rightarrow \infty} \frac{1}{\log t} \int_{1}^{t} a(\alpha s, f) \frac{d s}{s}=A
$$

uniformly in $\alpha \geq 1$.

Proof. The mapping $t \rightarrow a\left(e^{t}, f\right)$ is uniformly continuous since

$$
\begin{aligned}
\left|\frac{d}{d t}\left(a\left(e^{t}, f\right)\right)\right| & =\left|\frac{d}{d t}\left(\frac{1}{\psi\left(e^{t}\right)} \int_{0}^{e^{t}} f^{*}(s) d s\right)\right| \\
& =\left|-\frac{e^{t} \psi^{\prime}\left(e^{t}\right)}{\psi\left(e^{t}\right)} \frac{1}{\psi\left(e^{t}\right)} \int_{0}^{e^{t}} f^{*}(s) d s+\frac{e^{t} f^{*}\left(e^{t}\right)}{\psi\left(e^{t}\right)}\right| \\
& \leq 2\|f\|_{M_{\psi}} .
\end{aligned}
$$

The following theorem strengthens the result from [9, Corollary 3.9] in the case when $\psi \in \Omega$ satisfies (1.1).

Theorem 3.3. Let $\psi \in \Omega$ satisfy (1.1). A positive operator $T \in \mathcal{M}_{\psi}$ is Dixmier measurable if and only if there exists a limit in (1.9).

Proof. Suppose that $T \in \mathcal{M}_{\psi}$ is a Dixmier measurable positive operator; that is, $\operatorname{Tr}_{\omega}(T)=A$ for every Dixmier trace $\operatorname{Tr}_{\omega}$ on $\mathcal{M}_{\psi}$. According to Remark 1.1, we have $\tau_{\omega}(\mu(T))=A$ for every Dixmier functional $\tau_{\omega}$ on $M_{\psi}$. Denote, for brevity, $f:=\mu(T)$. By Corollary 3.2 we have

$$
\lim _{t \rightarrow \infty} \frac{1}{\log t} \int_{1}^{t} a(\alpha s, f) \frac{d s}{s}=A
$$

uniformly in $\alpha \geq 1$.

Using the pinching theorem one can show that the assumption (1.1) implies that

$$
\lim _{t \rightarrow \infty} \frac{\psi(N t)}{\psi(t)}=1 \text { for every } N>0 .
$$

So, for any $N>0$ one can find $t_{0}=t_{0}(N)$ such that for every $t>t_{0}$ we have

$$
\frac{\psi(t)}{\psi(N t)} \geq 1-\frac{1}{N}
$$


By the definition of a limit superior, there exists $\alpha>t_{0}$ such that

$$
a(\alpha, f) \geq\left(1-\frac{1}{N}\right) \limsup _{t \rightarrow \infty} a(t, f) .
$$

Using (3.2) and (3.3), we have

$$
\begin{aligned}
a(s, f) & \geq \frac{1}{\psi(\alpha N)} \int_{0}^{\alpha} f^{*}(u) d u=\frac{\psi(\alpha)}{\psi(\alpha N)} a(\alpha, f) \\
& \geq\left(1-\frac{1}{N}\right)^{2} \limsup _{t \rightarrow \infty} a(t, f)
\end{aligned}
$$

for every $s \in[\alpha, \alpha N]$.

Hence,

$$
\frac{1}{\log N} \int_{1}^{N} a(\alpha s, f) \frac{d s}{s}=\frac{1}{\log N} \int_{\alpha}^{\alpha N} a(s, f) \frac{d s}{s} \geq\left(1-\frac{1}{N}\right)^{2} \limsup _{t \rightarrow \infty} a(t, f) .
$$

Letting $N \rightarrow \infty$ and applying (3.1), we obtain

$$
A \geq \limsup _{t \rightarrow \infty} a(t, f) .
$$

Similarly one can prove that

$$
A \leq \liminf _{t \rightarrow \infty} a(t, f)
$$

and, therefore,

$$
\lim _{t \rightarrow \infty} a(t, f)=A .
$$

The converse implication is trivial.

Let us consider the Marcinkiewicz space $\mathcal{M}_{\psi}$ with $\psi(t)=2^{\sqrt{\log _{2}(1+t)}}-1$. It is easy to see that $\psi \in \Omega$ satisfies (1.1). Hence, $\mathcal{M}_{\psi}$ admits non-trivial Dixmier traces. A direct computation shows that $\psi(t)=2^{\sqrt{\log _{2}(1+t)}}-1$ does not satisfy (1.8).

The following theorem provides an example of a positive operator $T_{0} \in \mathcal{M}_{\psi}$ which is Connes-Dixmier measurable; however, it is not Dixmier measurable.

Theorem 3.4. Let $\psi(t)=2^{\sqrt{\log _{2}(1+t)}}-1$. There exists a positive Connes-Dixmier measurable operator $T_{0} \in \mathcal{M}_{\psi}$ such that the limit in (1.9) does not exist.

Proof. Let $T_{0}$ be such that

$$
\mu\left(T_{0}\right)=\sup _{k \geq 0} 2^{k-k^{2}} \chi_{\left[0,2^{k^{2}}\right)} .
$$

We set $f_{0}:=\mu\left(T_{0}\right)$. We obtain for every $2^{n^{2}} \leq t<2^{(n+1)^{2}}$,

$$
\begin{aligned}
a\left(t, f_{0}\right) & =\frac{1}{\psi(t)}\left(\int_{0}^{2^{n^{2}}} f(s) d s+\left(t-2^{n^{2}}\right) f_{0}(t)\right) \\
& =\frac{1}{\psi(t)}\left(\sum_{k=1}^{n} \int_{2^{(k-1)^{2}}}^{2^{k^{2}}} 2^{k-k^{2}} d s+t f_{0}^{*}(t)+O(1)\right) \\
& =\frac{2^{n+1}}{2^{\sqrt{\log _{2} t}}+\frac{t f_{0}^{*}(t)}{\psi(t)}+o(1) .}
\end{aligned}
$$


It is easy to see that $a\left(\cdot, f_{0}\right)$ is uniformly bounded and, so, $f_{0} \in M_{\psi}$. Hence, $T_{0} \in \mathcal{M}_{\psi}$.

By Lemma 1.2, for every dilation invariant generalized limit $\omega$ on $L_{\infty}$ that satisfies (1.6) we have

$$
\omega\left(\frac{t f^{*}(t)}{\psi(t)}\right)=0 \quad \text { for every } \quad f \in M_{\psi} \text {. }
$$

Denote by

$$
x(t):=\sum_{n=0}^{\infty} 2^{n-\sqrt{\log _{2} t}} \chi_{\left[2^{\left.n^{2}, 2^{(n+1)^{2}}\right)}\right.}(t) .
$$

We conclude from (3.4) and (3.5) that $\tau_{\omega}(f)=2 \cdot \omega(x)$ for every dilation invariant generalized limit $\omega$ on $L_{\infty}$ satisfying (1.6).

For every $2^{n^{2}} \leq t<2^{(n+1)^{2}}$, we have

$$
(M x)(t)=\frac{1}{\log t}\left(\sum_{k=0}^{n-1} \int_{2^{k^{2}}}^{2^{(k+1)^{2}}} 2^{k-\sqrt{\log _{2} s}} \frac{d s}{s}+\int_{2^{n^{2}}}^{t} 2^{n-\sqrt{\log _{2} s}} \frac{d s}{s}\right) .
$$

Since

$$
\int 2^{-\sqrt{\log _{2} s}} \frac{d s}{s}=-2 \cdot 2^{-\sqrt{\log _{2} s}}\left(\sqrt{\log _{2} s}+\frac{1}{\log 2}\right)+C
$$

and

$$
\int_{2^{n^{2}}}^{t} 2^{n-\sqrt{\log _{2} s}} \frac{d s}{s} \leq \int_{2^{n^{2}}}^{2^{(n+1)^{2}}} 2^{n-\sqrt{\log _{2} s}} \frac{d s}{s}=n+\frac{1}{\log 2}=o(\log t),
$$

we have

$$
\begin{aligned}
(M x)(t) & =\frac{-2}{\log t}\left(\sum_{k=0}^{n-1} 2^{k}\left(2^{-k-1}\left(k+1+\frac{1}{\log 2}\right)-2^{-k}\left(k+\frac{1}{\log 2}\right)\right)\right)+o(1) \\
& =\frac{2}{\log t} \sum_{k=0}^{n-1} \frac{k}{2}+o(1) \\
& =\frac{1}{2 \log 2}+o(1) .
\end{aligned}
$$

Hence, $\lim _{t \rightarrow \infty}(M x)(t)=\frac{1}{2 \log 2}$ and, therefore, $\tau_{\omega}(f)=\frac{1}{\log 2}$ for every ConnesDixmier functional $\tau_{\omega}$. Consequently, $T_{0}$ is a Connes-Dixmier measurable operator.

However, direct computation shows that

$$
\limsup _{t \rightarrow \infty} a\left(t, f_{0}\right) \geq \lim _{n \rightarrow \infty} a\left(2^{n^{2}}, f_{0}\right)=2
$$

and

$$
\liminf _{t \rightarrow \infty} a\left(t, f_{0}\right) \leq \lim _{n \rightarrow \infty} a\left(2^{(n+1 / 2)^{2}}, f_{0}\right)=\sqrt{2} .
$$

We conclude that $a\left(\cdot, f_{0}\right)$ has no limit at infinity and, so, a limit in (1.9) does not exist. 


\section{REFERENCES}

1. A. Carey, J. Phillips, F. Sukochev, Spectral flow and Dixmier traces, Adv. Math. 173 (2003), no. 1, 68-113. MR 1954456 (2004e:58049)

2. A. Carey and F. Sukochev, Dixmier traces and some applications to noncommutative geometry (Russian), Uspehi. Mat. Nauk 61 (6) (2006), 45-110. English translation in Russian Math. Surveys 61 (6) (2006), 1039-1099. MR2330013 (2009i:46132)

3. A. Carey, A. Rennie, A. Sedaev and F. Sukochev, The Dixmier trace and asymptotics of zeta functions, J. Funct. Anal. 249 (2007), no. 2, 253-283. MR2345333(2010i:46095)

4. A. Connes, Noncommutative Geometry, Academic Press, San Diego, 1994 . MR 1303779 (95j:46063)

5. J. Dixmier, Existence de traces non normales, C. R. Acad. Sci. Paris 262 (1966), A1107-A1108. MR.0196508 (33:4695)

6. P. Dodds, B. de Pagter, A. Sedaev, E. Semenov and F. Sukochev, Singular symmetric functionals, Zap. Nauchn. Sem. S.-Peterburg. Otdel. Mat. Inst. Steklov. (POMI) 290 (2002), Issled. po Linein. Oper. i Teor. Funkts. 30, 42-71 (Russian). English translation in J. Math. Sci. (N. Y.) 124 (2) (2004), 4867-4885. MR1942537 (2004a:46031)

7. P. Dodds, B. de Pagter, E. Semenov and F. Sukochev, Symmetric functionals and singular traces, Positivity 2 (1998), no. 1, 47-75. MR:1655756(99m:46072)

8. N.J. Kalton, A. Sedaev and F. Sukochev, Fully symmetric functionals on a Marcinkiewicz space are Dixmier traces, Adv. Math. 226 (2011), no. 4, 3540-3549. MR2764897(2012c:47057)

9. S. Lord, A. Sedaev and F. Sukochev, Dixmier traces as singular symmetric functionals and applications to measurable operators, J. Funct. Anal. 224 (2005), no. 1, 72-106. MR.2139105 (2006e:46065)

10. S. Lord and F. Sukochev, Measure Theory in Noncommutative Spaces, SIGMA 6 (2010), 072, 36 pp. MR2725011 (2012d:46149)

11. A. Pietsch, Dixmier Traces of Operators on Banach and Hilbert Spaces, to appear in Math. Nachr.

12. A. Pietsch, Shift-invariant functionals on Banach sequence spaces, preprint.

13. A. Pietsch, Connes-Dixmier Traces Versus Dixmier Traces, preprint.

14. A. Sedaev, Geometrical and topological aspects of interpolation spaces of Petre's K-method, Thesis, 2010 (in Russian).

15. F. Sukochev, A. Usachev and D. Zanin, Generalized limits with additional invariance properties and their applications to noncommutative geometry, submitted manuscript.

16. F. Sukochev and D. Zanin, Traces on symmetrically normed operator ideals, to appear in J. Reine Angew. Math.

School of Mathematics and Statistics, University of New South Wales, Sydney, NSW 2052, Australia

E-mail address: f.sukochev@unsw.edu.au

School of Mathematics and Statistics, University of New South Wales, Sydney, NSW 2052, Australia

E-mail address: a.usachev@unsw.edu.au

School of Mathematics and Statistics, University of New South Wales, Sydney, NSW 2052, Australia

E-mail address: d.zanin@unsw.edu.au 